\title{
Estrategias argumentativas para mejorar el pensamiento crítico en estudiantes universitarios
}

\author{
Luis Reynaldo Costábalos Acosta \\ luisrey_cosac2012@hotmail.es \\ https://orcid.org/0000-0002-9704-4821 \\ Universidad César Vallejo, Piura - Perú \\ César Balladares Atoche \\ cesarballadares@hotmail.com \\ https://orcid.org/0000-0001-8242-7742 \\ Universidad César Vallejo, Piura - Perú \\ Lucía Espinoza Cedillo \\ espinozacedillo@hotmail.com \\ https://orcid.org/0000-0001-7217-215X \\ Universidad César Vallejo, Piura - Perú
}

\section{RESUMEN}

La investigación tuvo como objetivo general Determinar las estrategias argumentativas para mejorar el pensamiento crítico en estudiantes de la Universidad Técnica de Babahoyo Extensión Quevedo, 2021, el enfoque fue cuantitativo de tipo básica, con diseño no experimental, transversal. Los datos se recogieron utilizando la técnica de la encuesta y como instrumento el cuestionario, aplicado a una muestra de 60 estudiantes de la Universidad Técnica de Babahoyo Extensión Quevedo, la confiabilidad es muy alta, según el Alfa de Cronbach. Los resultados determinaron que las estrategias argumentativas influyen positivamente en la mejora del pensamiento crítico de estudiantes, la tabla 3 evidencia que el 33\% se encuentra en nivel medio; de la misma manera los niveles de las dimensiones interpretación, análisis y evaluación, demuestran que se encuentran en nivel medio en $52 \%, 55 \%$ y $78 \%$, respectivamente.

Palabras clave: estrategias argumentativas; pensamiento crítico; interpretación análisis y evaluación 


\title{
Argumentative strategies to improve critical thinking in university students
}

\begin{abstract}
The general objective of the research was to determine the argumentative strategies to improve critical thinking in students of the Technical University of Babahoyo Extension Quevedo, 2021, the approach was quantitative of a basic type, with a non-experimental, cross-sectional design. The data were collected using the survey technique and as an instrument the questionnaire, applied to a sample of 60 students from the Technical University of Babahoyo Extension Quevedo, the reliability is very high, according to Cronbach's Alpha. The results determined that argumentative strategies positively influence the improvement of critical thinking in students. Table 3 shows that $33 \%$ are at an intermediate level; In the same way, the levels of the interpretation, analysis and evaluation dimensions show that they are at an average level in 52\%, 55\% and $78 \%$, respectively.
\end{abstract}

Keywords: argumentative strategies; critical thinking; interpretation; analysis and evaluation

Artículo recibido: 30 noviembre. 2021 Aceptado para publicación: 29 diciembre 2021 Correspondencia: luisrey_cosac2012@hotmail.es Conflictos de Interés: Ninguna que declarar 


\section{INTRODUCCIÓN}

Es importante resaltar que la sociedad actual presenta serias dificultades para poder generar pensamiento crítico, lo que dificulta la capacidad de solucionar las crisis emitidas por diferentes situaciones, impidiendo severamente convivir pacíficamente entre sus iguales. Si la formación del pensamiento crítico viene determinada desde la infancia, se puede alcanzar niveles óptimos que faciliten dicha situación.

Considerando las exigencias educativas y sociales que demanda el contexto mundial, además los avances científicos y tecnológicos, frente a los problemas identificados en el devenir histórico, como también geográficos y económicos, en espacios tanto macros como micros, se comprende que las prácticas de enseñanza rutinarias y mecánicas, bajo paradigmas memorísticos, hoy en día pierden vigencia, debido a que no hace posible el logro de competencias y habilidades básicas o necesarias requeridas por los estudiantes en la actualidad.

A nivel internacional, se enfatiza la atención a la problemática, asumiendo el compromiso establecido por la UNESCO, como el objetivo de la educación para todos, sin embargo, aun existiendo directrices, que direccionan lineamientos del pensamiento crítico, se visualiza que se persiste, la aplicación de estrategias rutinarias, que no promueven prácticas del conocimiento acorde a las exigencias sociales, sin considerar la diversidad geográfica, ni las distintas realidades de los estudiantes, evidenciadas en la interactuación en las aulas de clases.

En Barranquilla, Colombia, el 90\% de estudiantes de la Universidad de la Costa, presentan bajo nivel de pensamiento Automático, en la Universidad del Atlántico el 30\%, presentó bajos niveles en el mismo pensamiento, lo que permite deducir que no tuvieron la capacidad de comprensión y extracción de las ideas del texto trabajado. En el estudio realizado, se observa que la muestra de estudio, presentan bajos niveles en el desarrollo del pensamiento crítico. (Steffens, 2017).

El Ecuador no es ajeno a esta problemática, lo que ha permitido que se constituya como un freno importante que lo mantiene aún en la lista de países del tercer mundo, nos referimos a la preocupante situación de su educación, en gran parte por la presencia de bajos niveles de pensamiento crítico. En las programaciones curriculares donde se consideran habilidades de pensamiento como: inferir, argumentar, opinar, comparar, en 
un $42.9 \%$ son poco satisfactorio, de la misma manera los estudiantes en un $57.15 \%$ demuestran que estas habilidades no han sido desarrolladas. (Illescas, 2011).

Es importante también mencionar que, por muchas y diversas razones, es necesario el interés para promover el pensamiento crítico; entre ellas es la ausencia de habilidades del pensamiento y la capacidad de criticidad que debe ir en crecimiento en el sujeto, para actuar en concordancia con el mundo moderno que lo demande; así mismo, el poder participar con actitud crítica y democrática, en la realidad actual.

Se formula la pregunta de investigación: ¿En qué medida las estrategias argumentativas mejoran el pensamiento crítico en estudiantes universitarios? De la misma manera se planteó el objetivo general: Determinar que las estrategias argumentativas mejorar el pensamiento crítico en estudiantes.

De la revisión de trabajos previos, encontramos a Roca (2016) España, en su trabajo titulado "Desarrollo del pensamiento crítico a través de diferentes metodologías docentes en el grado de enfermería", presentado en la Universidad Autónoma de Barcelona. Estudio con 65 estudiantes, asignatura enfermería clínica de segundo grado. Su objetivo, valorar el desarrollo de la competencia del pensamiento crítico a partir de la implementación de diferentes estrategias metodológicas de enseñanza aprendizaje, enfoque fue mixto, con diseño añadido concurrente de varios niveles (DIACNIV). Los instrumentos empleados fueron cuestionario PENCRISAL, como técnica observación en el aula, diario de campo, cuestionarios post intervención. Las conclusiones fueron: el pensamiento crítico está relacionado a la reflexión, resolución de problemas, toma de decisiones y razonamiento.

Asimismo, se considera a Aguila (2016) presenta "Habilidades y estrategias para el desarrollo del Pensamiento crítico y creativo en estudiantes de la universidad de sonora", México, estudio desarrollado con muestra de 217 alumnos. presenta como objetivo, conocer en qué medida las herramientas y el lenguaje del pensamiento crítico desarrollan formas de aprendizaje, en la escuela y en la vida cotidiana, desde un enfoque cualitativo y cuantitativo, disponiendo de diseño exploratorio-descriptivo, el instrumento utilizado es cuestionario, concluye que carecen de elementos necesarios para la definición de conceptos, es decir los estudiantes ponen en práctica algunos estándares claros, con profundidad, justicia y lógica. 
Así también, Huamán (2016), Perú, sobre “Argumentación como estrategia didáctica para desarrollar la competencia oral en estudiantes del I ciclo de Derecho", universidad César Vallejo, 27 educandos como muestra, tanto para el grupo de control y experimental. Tuvo como objetivo demostrar la aplicabilidad de la estrategia de la argumentación y su influencia en el desarrollo de la competencia oral de los futuros abogados, el diseño cuasi experimental, instrumento una rúbrica. Concluyendo que el grupo experimental, mejoro el nivel de competencia oral de los estudiantes, obteniendo nivel regular, bueno y muy bueno con 48\%, 33\% y 19\%; en cambio, el grupo de control, sostuvo los niveles, deficiente y regular, el 15\% logró el nivel bueno. Demostrando mayor fluidez, cuando usa argumentos coherentes a la competencia oral.

De la misma manera Remache (2017) Ecuador, investigó sobre el "Pensamiento crítico y habilidades comunicativas básicas articulados al perfil de ingreso para la universidad". La muestra fue de 375 , estudiantes de bachillerato y estudiantes de dos universidades. Presenta como objetivo analizar el pensamiento crítico y su relación con las habilidades comunicativas de los estudiantes que ingresan a la universidad, con diseño exploratorio descriptivo, apoyado en el cuestionario como instrumento. Las conclusiones fueron: Alumnos de bachillerato ofrecen nivel de respuesta media, por debajo de los universitarios, situación que permite entender que la madurez cognitiva y el proceso de formación universitaria mejoría las dimensiones evaluadas; en segundo nivel, por lo previsto su mayoría, se encontró en un nivel menor al semestre superior, accionar que permite acceder a consolidar la formación como proceso, aportando al desarrollo de las habilidades del pensamiento.

De acuerdo a Parra, (2018), Ecuador, maestría, en su investigación titulada: Desarrollo de habilidades del pensamiento crítico y su incidencia en la fluidez verbal en los estudiantes de la facultad de filosofía de la universidad de Guayaquil, tiene como objetivo incrementar las habilidades mediante la guía de estrategias, metodología aplicada 20 docentes y 206 alumnos, facultad de filosofía, universidad de Guayaquil, utilizando la encuesta; concluyéndose que, los educadores deben promover la argumentación y criticidad reflexiva en el espacio educativo, debido a la falta de utilización de estrategias que motiven el pensamiento crítico, porque las intervenciones orales son calificadas como regulares, sin considerar que cotejan puntos de vista personales y ajenos. 
De igual forma Palma (2017). Ecuador, Pensamiento crítico desarrolla destrezas de reading y speaking del idioma inglés, en estudiantes de noveno de básica, de la unidad educativa "Liceo Policial". Nos refiere que el objetivo fue describir la manera que el pensamiento crítico se constituye en alternativa para desarrollar destrezas, tipo de enfoque fue cuali-cuantitativo, nivel descriptivo. empleó la encuesta, el cuestionario para recoger datos, muestra fueron 33 estudiantes y 2 docentes de inglés, los resultados evidencian que los docentes, pocas veces, realizan actividades para estimular el desarrollo del pensamiento crítico, entonces, previniendo dificultades para interpretar e analizar, responder preguntas, aclarando opiniones, e identificar ideas principales y secundarias.

El trabajo se sustenta en el aprendizaje significativo, teoría de David Ausubel, sostiene que el ser humano aprende de lo que tiene sentido y lógica; lo que le conviene e interesa para su accionar diario, previamente debe contar con cantidad básica de información, para relacionar los nuevos conocimientos, con los que ya tiene, el profesor es mediador entre lo nuevo y lo antiguo. (Ausubel 1983).

De la misma manera teoría de procesamiento de la información, sustentada Gagné et. al., manifiestan que la inteligencia humana desarrolla capacidad de innovación, crean conocimiento, desarrolla operaciones simbólicas de codificación, comparación. Además, consideran al ser humano como procesador de información, comparándolo con el funcionamiento de un computador, de lo cual surgen antagónicas posiciones, una limitada increíble equivalencia; y otra fuerte que admite una equivalencia funcional entre ambos sistemas.

Como lo expresa la toría cognitiva de Lonergan. Las personas disponen de una variedad de operaciones mentales internas, no observables, contribuyendo al procesamiento real. El alumno realiza acciones mentales, de igual el educador como toda persona, se puede mencionar ciertas operaciones: preguntar, recordar, ver, oír, imaginar, sentir, oler, gustar, comparar, memorizar, repetir, entender, analizar, concebir, explicar, formular, escribir, hablar, hipotetizar, calcular, conceptualizar, juzgar, recoger evidencias, razonar, reflexionar, ordenar y ponderar las evidencias, argumentar, decidir, deliberar, evaluar. Al utilizar una operación mental, por el sujeto, se convierte en habilidad con destreza mentales. (Lonergan 1999, citado por De la Chaussée).

En este, enfoque la comprensión textual, McKoon \& Ratcliff. (1998) mirado como el mesurado desenlace de procesos inferenciales, mecanismos, estrategias lingüísticas y 
cognitivas, el leyente, activa de forma interactiva e estratégica. Paris et al. (1994), Las diversas dimensiones, se describen identificándose e interactuando en la comprensión de textos, componentes, como la cohesión sintáctica y semántica; la macro - microestructura; la concepción de semejantes, caracterizando a ellas.

Perelman, et al. (2001). Las estrategias argumentativas, es toda acción utilizada con eficacia en los discursos, de manera consiente e intencional utilizan los hablantes o el escritor, para superar el convencimiento al destinatario en momentos comunicativos donde allá argumentación. (Perelman, 2001; Olbrechts-Tyteca, 1989; Plantin, 1998; Silvestri, 2001). Continuando con la investigación, tenemos los principales tipos de argumentación: Inductiva, fundamentada, en conjeturar, premisas generadas por argumentos, aprovechando la mejora de la conclusión; deductiva, extrae conclusiones de premisas que son comprobables y verificables; abductiva, muy parecida a la argumentación inductiva por extraer conclusiones, partiendo de premisas; analogía, es con exactitud el tema, aclarando evidencias que producen conclusiones más o menos lógica. (Toulmin et al., 1979, citado por Díaz 2006).

Estrategias argumentativas, vista como el conjunto de acciones mentales, desplegadas de los procesos de enseñanza-aprendizaje, para que el sujeto aprenda, buscando, formas de interacción y de trabajo mentalmente. En algunos planteamientos se deben usar ejemplos, citas, refranes, prosopopeyas, metáforas, analogías, explicaciones, descripciones, inferencias, explicaciones causales, comparaciones, deducciones, explicaciones causales. Estas estrategias se ponen en juego, para conseguir un propósito. (Barker, 1991, \& Guétmanova, 1989).

Competencia lectora, definida como la capacidad de un individuo para reflexionar, comprender, utilizar e interactuar con diversos textos escritos, que promuevan alcanzar metas, desarrollando sus conocimientos, potenciales, participando en la sociedad. En el año 2000, como área base fueron: Escalas de lectura y competencia lectora, dividiéndose en cinco niveles de destrezas y conocimientos. La primordial ventaja de este enfoque, es provechoso describiendo el número previsto puede hacer asociado los ejercicios a diferentes niveles de dificultad. (Álvarez, 2001).

Igual modo la variable dependiente, pensamiento crítico, no existe definición universal del pensamiento crítico dado el carácter complejo y multidimensional del término. Tamayo (2009) sostiene: El pensamiento crítico exige formas nuevas de entender las 
interrelaciones entre los saberes existentes en las aulas, siendo una razón, la dificultad que presentan los educandos para utilizar conocimientos, explicar y comprender fenómenos cotidianos, derivado de las actividades didácticas tradicionales, que direccionan a la aprehensión de conceptos básicos, relacionamos al funcionamiento de máquinas que usan a diario aplicando los principios de su funcionamiento, como incapacidad para entender.

\section{ESTRATEGIAS METODOLÓGICAS O MATERIALES Y MÉTODOS}

La investigación estuvo orientada con enfoque cuantitativo, según Valderrama (2013), ya que se recogieron y utilizaron datos que sirvieron para proyectar acciones sobre estrategias argumentativas. La investigación es básica (CONCYTEC 2018) con diseño no experimental, del tipo transversal, además no hay manipulación de variables. Por lo general, su utilidad está dada como el inicial acercamiento al problema investigado (Hernández et al., 2014). Nivel descriptivo, fundamentado en el análisis del problema, detallando problemas de interés, al mismo tiempo edifica a indagaciones que necesitan una altitud descriptiva avanzado. (Muntané, 2010).

La variable independiente, estrategias argumentativas, consiste en brindar un conjunto de elementos o herramientas para propiciar elevar la competencia del nivel crítico, con razones en apoyo de una conclusión. Los argumentos son intencionalidades de apoyo, ciertas opiniones con razones". (Weston, 2006)

Asimismo, la variable dependiente: Pensamiento crítico entendida como proceso consciente y deliberado, usado para la interpretación y evaluación, de los testimonios como experiencia, que direccionan acciones y creencias, mediante un conjunto de actitudes y habilidades. (Remache, 2017).

La población fueron 698 estudiantes de la Universidad Técnica Babahoyo, Extensión Quevedo - Ecuador. La muestra fue de 60 estudiantes, cuya elección fue mediante el muestreo no probabilístico, sin utilizar fórmula matemática, a conveniencia del autor

Se utilizó la encuesta como técnica y el cuestionario como instrumento, por su pertinencia y naturaleza de la investigación, solamente utilizando la virtualidad, siendo de mucha utilidad en momentos de la virtualidad. Para la confiabilidad, los datos se procesaron por el método de consistencia interna y el estadístico Alfa de Cronbach, resultando de magnitud Muy alta, ya que se encuentra resulta 0,832. Los resultados fueron analizados 
descriptivamente, presentándolos estadísticamente, en cuadros, tablas y figuras, con frecuencias y porcentajes, cada uno con sus respectivas interpretaciones.

\section{RESULTADOS Y DISCUSIÓN}

Los resultados fueron obtenidos con la aplicación del cuestionario, de manera virtual, dadas las circunstancias de la pandemia, las interrogantes están dirigidas a la consecución del objetivo general, obteniendo los siguientes resultados:

\section{Objetivo general:}

- Determinar las estrategias argumentativas para mejorar el pensamiento crítico en estudiantes.

Tabla 1 Niveles de la variable pensamiento crítico

\begin{tabular}{ccc}
\hline & $\mathbf{f}$ & $\mathbf{\%}$ \\
\hline Alto & 38 & 64 \\
Medio & 20 & 33 \\
Bajo & 2 & 3 \\
TOTAL & $\mathbf{6 0}$ & $\mathbf{1 0 0}$ \\
\hline
\end{tabular}

Interpretación: La tabla 1, muestra los niveles de la variable pensamiento crítico, donde se aprecia que el $33 \%$ se encuentra en nivel medio, el $3 \%$ en nivel bajo y el $64 \%$ nivel alto. Lo que indica que el pensamiento crítico se ubica nivel medio, por tanto, se requiere de estrategias para mejorar.

\section{Objetivo especifico 1}

- Determinar el nivel de interpretación

Tabla 2 Niveles de la dimensión interpretación

\begin{tabular}{ccc}
\hline & $\mathbf{f}$ & $\mathbf{\%}$ \\
\hline Alto & 25 & 41 \\
Medio & 31 & 52 \\
Bajo & 4 & 7 \\
TOTAL & $\mathbf{6 0}$ & $\mathbf{1 0 0}$ \\
\hline
\end{tabular}

Interpretación: En la tabla 2, se observa los niveles de la dimensión interpretación, donde se aprecia que el $52 \%$ se visualiza en nivel medio, el $41 \%$ en nivel alto y el $7 \%$ nivel bajo. Lo que indica que la dimensión interpretación se ubica nivel medio, por tanto, se requiere de estrategias para mejorar. 


\section{Objetivo especifico 2.-}

- Determinar el nivel de análisis

Tabla 3: Niveles de la dimensión análisis

\begin{tabular}{ccc}
\hline & $\mathbf{f}$ & \% \\
\hline Alto & 25 & 42 \\
Medio & 33 & 55 \\
Bajo & 2 & 3 \\
TOTAL & $\mathbf{6 0}$ & $\mathbf{1 0 0}$ \\
\hline
\end{tabular}

Interpretación: En la tabla 3, se observa los niveles de la dimensión análisis, donde se aprecia que el 55\% se encuentra en nivel medio, el $42 \%$ en nivel alto y el $3 \%$ nivel bajo. Lo que indica que el análisis se ubica nivel medio, por tanto, se requiere de estrategias para mejorar

\section{Objetivo especifico 3.-}

- Determinar el nivel de evaluación

Tabla 4: Niveles de la dimensión evaluación

\begin{tabular}{ccc}
\hline & $\mathbf{f}$ & $\boldsymbol{\%}$ \\
\hline Alto & 10 & 17 \\
Medio & 47 & 78 \\
Bajo & 3 & 5 \\
TOTAL & $\mathbf{6 0}$ & $\mathbf{1 0 0}$ \\
\hline
\end{tabular}

Interpretación: En la tabla 4, se observa los niveles de la dimensión evaluación, donde se aprecia que el $78 \%$ se encuentra nivel medio, el 5\% nivel bajo y el 17\% nivel alto. Lo que indica que la evaluación se ubica nivel medio, por tanto, se requiere de estrategias para mejorar.

Cada ser humano tiene una forma de pensar, de una forma específica, cada alumno tiene grandes diferencias de adquirir el pensamiento crítico, los cuales están relacionados a diversos procesos.

Para el objetivo general, los resultados determinan que las estrategias argumentativas mejoran el pensamiento crítico en estudiantes universitarios. En la tabla 1, se observa que para la variable pensamiento crítico, donde se aprecia que el $33 \%$ se encuentra en nivel medio, por tanto, se requiere de nuevas estrategias que ayuden a reflexionar al estudiante 
y logren el nivel alto en su mayoría. El estudio encuentra similitud con la teoría del aprendizaje significativo, donde la persona aprende lo que tiene significado, lógica y sentido en su vida diaria. De la misma manera se sustenta en el trabajo de Facione (2007), el pensamiento crítico porque el sujeto desarrolla destrezas diversas de: interpretación, inferencia, análisis, explicación, autorregulación y evaluación, lo mismo que con congruencia, pautas de coherencia, exactitud, precisión y fortaleza lógica basada en las inferencias reales o suposiciones. De igual modo Parra, (2018), concluye que el maestro motive al alumno a la crítica y reflexión argumentativa, al expresarse verbalmente o escrita, no continuamente demuestran lo comprendido, pues necesitan estrategias que incrementen el vocabulario, como el saber utilizar antónimos y sinónimos, en sus argumentaciones. De tal manera Cuenca (1995), dice que las estrategias argumentativas, tienen como función convencer a la audiencia en sentido general, para ello se debe considerar tres funciones básicas: La expresiva, la función apelativa y la representatividad que tiene que ver con el contexto.

OE 1.- Los hallazgos obtenidos para determinar los niveles de la dimensión interpretación en estudiantes. Se evidencia en la tabla 2, que esta dimensión, presenta el $41 \%$ se encuentra en nivel alto, lo que indica que, la dimensión interpretación se ubica en nivel alto, seguido $52 \%$ se encuentra en nivel medio, donde requiere de mejora de estrategias argumentativas para lograr estandarizar casi en su totalidad alcancen el nivel alto. Sosteniéndose en el estudio de Soler (1995), indica, que la interpretación lo que busca es la confluencia del hombre con su verdad, así también la captura del ser humano y su inconsciente, tiene más bien una función divisoria, de límite, de distancia que favorece el encuentro de sí mismo con su deseo. Del mismo modo, Villarini (2006), nos dice que las personas poseen actitudes mentales y operaciones para resolver problemas, definiendo los medios para la toma de decisiones. Tomando la propuesta, Del Caño (1999), manifiesta, la argumentación puede tener dos determinaciones: primero, convencer al destinatario que adopte determinados comportamientos, actitud, de tal manera que comparta ideas; segundo, incitar al consignatario para que asuma una idea o realice una acción, apelando a sus sentimientos. Si el docente toma como referente los aportes de estos autores va a planificar estrategias que ayuden a desarrollar la capacidad de interpretación. 
O.E 2.- Según los hallazgos para determinar el nivel de la dimensión análisis en estudiantes, en la tabla 3, se aprecia que el 55\% se encuentra en nivel medio, por tanto, según Tesch (1990), del análisis se puede decir que estudia las razones últimas que provocan una determinada circunstancia, y proyecta su comportamiento en el futuro a partir de una descripción o incluirla. Por otro lado, Aguilar (1996); manifiesta que se debe tener en cuenta las tres características para un buen análisis debe ser atemporal, es de implicación y no deductiva, es general y no antropocéntrica, pues orienta a descubrir la lógica implícita. Según la Teoría de Procesamiento de la Información, nos indica que el ser humano debe realizar operaciones simbólicas como comparar, localizar, codificar y almacenar, demostrando su capacidad de innovación para crear conocimiento. Se coincide con el trabajo de Roca en España (2016) ya que entre sus conclusiones sostiene al pensamiento crítico, muestra estrecha relación con la resolución de problemas, meditación, razonamiento y la toma de decisiones. Es pertinente indicar que es necesario gestionar de manera adecuada las actividades curriculares para propiciar el análisis en los estudiantes.

OE 3.- Determinar los niveles de evaluación en estudiantes de la Universidad Técnica de Babahoyo Extensión Quevedo, 2021. En la tabla 4, se puede visualizar que el $78 \%$ se encuentra en el nivel medio, por lo visto se deben proveer estrategias argumentativas para mejorar la evaluación. Como indica MINEDUC (2020) la evaluación, debe contextualizarse y flexibilizarse, considerando la realidad del contexto. Se coincide con la investigación realizada en México por Aguila (2016) quien manifiesta que para valorar el pensamiento es necesario considerar los juicios propios o ajenos, que presenten criterios de exactitud, coherencia, claridad y pertinencia, con basamento reales $\mathrm{o}$ conjeturas para obtener conclusiones razonables. Por otra parte la investigación encuentra sustento en el trabajo de Parra (2018) Ecuador, manifiesta que los maestros deben promover criticidad, argumentación reflexiva y la crítica en el contexto escolar, evidenciando la falta de uso de estrategias por parte del educador, con frecuencia, estimulando el pensamiento crítico en las actividades, además la evaluación es homogénea, ya que se consideran regulares las participaciones orales de los estudiosos, aun cuando en realidad exteriorizan puntos de vista diversos, no confrontaciones, sino distintos argumentos. Por tanto, se debe promover y motivar, las actitudes empáticas, que favorezcan la comprensión de diversas situaciones para los estudiantes y sus familias. 
Presentando similitud en las teorías, entre aprendizaje significativo y procesamiento de la información, ya que queda demostrado que los seres humanos, sin excepción, actuamos y experimentamos diversas situaciones, como consecuencia de la práctica diaria, influidas por las relaciones sociales y del mundo circundante. Las interrelaciones sociales, a nivel familiar, amical, comunal, étnico o de cualquier índole, nos ofrece elementos, rasgos y caracteres, que se convierten en conocimientos

\section{CONCLUSIÓN O CONSIDERACIONES FINALES}

Se ha determinado que las estrategias argumentativas influyen en la mejora del pensamiento crítico en estudiantes universitarios. Lo que se evidencia en la tabla 1 , donde el 33\% se encuentra en nivel medio, haciéndose necesario la aplicación de nuevas estrategias que favorezcan la reflexión en los estudiantes y buscar ubicarse en nivel alto. Es pertinente indicar que se encuentran debilidades en el razonamiento con sentido lógico. Se necesita desarrollar competencias para promover las actitudes analíticas, inferenciales e interpretativas, enfatizando en la autorregulación individual.

Se determina que los niveles de la interpretación en estudiantes, en la tabla 2, se observa al $41 \%$ en nivel alto, quedando un $52 \%$ entre nivel medio, se hace evidente que se requiere aplicar estrategias argumentativas para lograr estandarizar al 100\% en nivel alto. Tomando en consideración el estudio de Soler (1995), donde señala que la interpretación busca encontrar al individuo con su verdad, como la captura de su inconsciente. Se coincide con Del Caño (1999), cuando manifiesta que la argumentación puede convencer al destinatario para adoptar comportamientos o actitudes determinadas, o persuadir para que ejecute una acción o asuma una postura, apelando a sus sentimientos. Los docentes deben planificar y desarrollar adecuadamente estrategias para propiciar la capacidad de interpretación.

Se determina que los niveles de la capacidad analítica de estudiantes, se observa que el $55 \%$ se encuentra en nivel medio (tabla 3). Tomando en consideración lo sostenido por Tesch (1990), se deben desarrollar actividades que estimulen la inferencia y deducción, para que el estudiante actúe adecuadamente, con capacidad crítica reflexiva en las diferentes circunstancias o momentos de su vida diaria. Roca (2016) también es coincidente al sostener que la reflexión, el razonamiento, ayudan a resolver problemas cotidianos y adoptar decisiones pertinentes; por ello, es oportuno indicar que se deben 
arribar a consensos institucionales para desarrollar actividades curriculares que propicien el análisis en los estudiantes.

Se ha determinado que los niveles de la evaluación, en estudiantes universitarios. La tabla 4, presenta al 78\% de los encuestados en nivel medio. El MINEDUC, en el año 2020, sostiene explícitamente que la evaluación, como proceso de recojo de evidencias, debe concebirse desde el contexto real, y considerar las peculiaridades de cada estudiante. Coincidentemente con Aguila (2016) se deben establecer criterios de claros, coherentes y pertinentes, donde se promueva la criticidad, argumentación reflexiva en el contexto escolar, lo que se deduce que los profesores no utilizan adecuadas estrategias para tal finalidad. Es evidente la necesidad de satisfacer la demanda estudiantil en lo que se refiere a las actividades para reflexionar y motivar, para la adopción de prácticas empáticas, con efectos positivos en las relaciones sociales, tanto en la familia y otros espacios.

\section{LISTA DE REFERENCIAS}

Abrami, P., Bernard, R.M., Borokhovski, A., Wade, A., Surkes, M.A., Tamin, R., \& Zhang,

D.

(2008).

Instructional interventions affecting critical thinking skills and dispositions: A stage 1 meta-analysis. Review of Educational Research, 78(4), 1102-1134. DOI: 10.3102/0034654314551063@ 2014 AERA. http://rer.aera.net

Aguila, E. (2016). Habilidades y estrategias para el desarrollo del Pensamiento crítico y creativo en alumnado de la Universidad de Sonora. (Tesis doctoral, Universidad de Extremadura)

Recuperado: http://dehesa.unex.es/bitstream/handle/10662/1774/TDUEX_2014_Aguila_More no.pdf? sequence $=1$

Alejo, L. (2017). El pensamiento crítico en estudiantes del grado de maestro/a en educación primaria desde la didáctica de las ciencias sociales. (Tesis doctoral, Universidad de Málaga, España). Recuperado de: https://riuma.uma.es/xmlui/bitstream/handle/10630/15997/TD_ALEJO_LOZAN $\underline{O}$ LLaura.pdf? sequence $=1$ \&isAllowed $=y$

Campaner, G., De Longhi, A. (2007). La argumentación en Educación Ambiental. Una estrategia didáctica para la escuela media. Recuperado de: http://reec.webs.uvigo.es/volumenes/volumen6/ART12_Vol6_N2.pdf 
Carrillo Guerrero, L.( 2007). Argumentación y argumento, revista signa, universidad de Granada, v.16 pp. 289-320, [pdf]. Recuperado en:

file:///C:/Users/HP/AppData/Local/Temp/Dialnet-

ArgumentacionYArgumento-2216764.pdf

Coello Caicedo, A. (2015). El Pensamiento crítico en el proceso de enseñanza aprendizaje de los estudiantes de educación general básica superior de la unidad educativa fiscomisional San Daniel Comboni de la ciudad de Esmeraldas, 20132014. [tesis maestria] https://repositorio.pucese.edu.ec/bitstream/123456789/392/1/COELLO\%20CAI CEDO\%20ANGELA\%20IRENE.pdf

De la Chaussée Acuña, M. (2009). Las estrategias argumentativas en la enseñanza y aprendizaje de la química. Educ. quím, vol.20, no.2, ciudad de México abr. 2009. Recuperado de: http://www.scielo.org. $m x / p d f / e q / v 20 n 2 / v 20 n 2 a 8 . p d f$

Díaz Alfonso, B., Soler Arias, A. Zavala Alexander, H. (2020), Estrategias Didácticas para Potenciar el Pensamiento Crítico desde la Comprensión Lectora, Universidad Santo Tomás Tunja. Recuperado de: https://repository.usta.edu.co/bitstream/handle/11634/30458/2020hernandozabal a.pdf? sequence $=5 \&$ isAllowed $=y$

Fernández, G. (2017). Argumentación y lenguaje jurídico. Recuperado de: https://archivos.juridicas.unam. $m x / w w w / b j v / l i b r o s / 7 / 3016 / 1 . p d f$

García Lorente, J. (2016) La ciencia de los principios y de las causas primeras en el libro primero de la Metafísica, Anales del Seminario de Historia de la Filosofía, universidad complutense de Madrid, España vol. 33, núm. 1, pp. 11-31.

http://www.redalyc.org/articulo.oa?id=361146065001.

Hernández, Fernandez y baptista, (2010). Metodología de la investigación (5a ed.).

México: Mc Graw Hill https://www.esupág.edu.pe/descargas/dep_investigacion/Metodologia\%20de\%20l a\%20investigaci\%C3\%B3n\%205ta\%20Edici\%C3\%B3n.pdf

Huamán, N. (2016). La argumentación como estrategia didáctica para el desarrollo de la competencia oral en estudiantes del I ciclo de Derecho, Universidad César Vallejo - Tarapoto. (Tesis doctoral, Universidad César Vallejo). Recuperado de: 
http://repositorio.ucv.edu.pe/bitstream/handle/UCV/13856/huaman_tn.pdf?sequ ence $=1$ \&isAllowed $=y$

Hurtado Mondoñedo, L. (2017). Review of the Definitions of Propositions and Statement in their Relationship with Mathematics, Rev. Digit. Invest. Docencia Univ. Vol. 11. $N^{\circ} .1$ Lima, 2017. http://dx.doi.org/10.19083/ridu.11.481

Illescas Ortega, J. (2011). Habilidades para el desarrollo del pensamiento crítico en el currículo del primer año, (Tesis de maestría, Universidad de Cuenca Ecuador). Recuperado en: http://dspace.ucuenca.edu.ec/handle/123456789/2787

Julieta Migdalek, M., Santibáñez Yáñez, C., Renata Rosemberg, C. (2013). Argumentative strategies in young children: An study of disputes during play in school contexts, revista signos. estudios de Lingüística issn 0718-0934, Chile. DOI: 10.4067/S0718-09342014000300005 • 47(86) 435-462

Makuc Sierralta, M., Larrañaga Rubio, E. (2013). Implicit theories about reading comprehension:

Exploratory study in university students, revista signos. estudios de linguiística, 2015 PUCV, Chile. pp. 48(87) 29-53. Recuperado en:

DOI: $10.4067 /$ S0718-09342015000100002 • 48(87) 29-53.

Ministerio de Educación, (2020). Instructivo para la evaluación estudiantil, toda una vida, Ecuador. Recuperado en: https://educacion.gob.ec/wpcontent/uploads/downloads/2020/09/Instructivo-para-evaluacion-de-losaprendizajes-Sierra-y-Amazonia-2020-2021.pdf

Muñoz, N. (2018). Estrategias argumentativas utilizadas por los estudiantes de la institución educativa Divino Niño de Tierra Grata (Chinú) en el análisis de hechos históricos culturales. Recuperado de: file:///C:/Users/Computer/Downloads/DialnetEstrategiasArgumentativasUtilizadasPorLosEstudiant-6860277.pdf

Navarro Ramírez, D. (2013). El proceso de observación: El caso de la práctica supervisada en inglés en la sede de occidente, universidad de Costa Rica, inter sedes: revista de las sedes regionales, v. xiv, $\mathrm{n}^{\circ} 28$, pp. 54-69. Recuperado http://www.redalyc.org/articulo.oa?id=66629446004

Palma, G.(2016). Pensamiento crítico en el desarrollo de las destrezas de reading y speaking del idioma inglés, en estudiantes de noveno de básica, de la Unidad 
Educativa "Liceo policial”, Quito, período 2016, [Tesis de maestría, universidad central del Ecuador]. Recuperado en: http://www.dspace.uce.edu.ec/handle/25000/16409

Parra, Salazar I. (2015). Desarrollo de habilidades del pensamiento crítico y su incidencia en la fluidez verbal en los estudiantes de la facultad de filosofía de la universidad de guayaquil, propuesta: guía de estrategias. (Tesis doctoral, Universidad de Guayaquil, Ecuador). Recuperado de: http://repositorio.ug.edu.ec/bitstream/redug/2035/1/TESIS\%20MAESTRIA\%20D EFINITIVA\%20ULTIMA.pdf

Remache Bunci, M. (2017). Pensamiento crítico y habilidades comunicativas básicas articulados al perfil de ingreso para la universidad. (Tesis doctoral, Universidad de Extremadura) Recuperado de: file:///C:/Users/Computer/Desktop/ecuador\%20tesis/pensamiento\%20criticohabilidades\%20comunicativas.pdf

Rodríguez Frias, M. y Flotts de los Hoyos, M. (2019), Definición del referente de la evaluación y desarrollo del marco de especificaciones, Instituto nacional para la evaluación México, https://www.inee.edu.mx/wpcontent/uploads/2019/08/P2A353.pdf.

Salazar, F., Samayoa, V., López, G. (2015). El pensamiento reflexivo en la formación del futuro profesor. Recuperado de: https://www.redalyc.org/pdf/461/46142596020.pdf

Sanchez Carlessi, H., Reyes Romero, C. y Mejia Sáenz, K. (2018). Manual de Términos en Investigación científica, tecnológica y humanística, universidad Ricardo Palma, Lima. Perú. Recuperado https://www.urp.edu.pe/pdf/id/13350/n/libro-manual-de-terminos-eninvestigacion.pdfhttp://ve.scielo.org/scielo.php?script=sci_arttext\&pid=S0459$\underline{2832008000200004}$

Vara, A. (2012). 7 pasos para una tesis exitosa. Desde la idea inicial hasta la sustentación. Un método efectivo para las ciencias empresariales. Instituto de Investigación de la Facultad de Ciencias Administrativas y Recursos Humanos. Universidad de San Martín de Porres. Lima 
https://www.administracion.usmpág.edu.pe/investigacion/files/7-PASOS-PARA-UNATESIS-EXITOSA-Desde-la-idea-inicial-hasta-la-sustentaci\%C3\%B3n.pdf

Varela, J. (2015), Algunas referencias sobre la interpretación: desde Freud a Lacan. VII Congreso Internacional de Investigación y Práctica Profesional en Psicología XXII Jornadas de Investigación XI Encuentro de Investigadores en Psicología del MERCOSUR. Facultad de Psicología - Universidad de Buenos Aires. Disponible en: https://www.aacademica.org/000-015/861.[pdf].

Vera L. (2010). Formación Continuada y Varias Voces del Profesorado de Educación Infantil de Blumenau: Una Propuesta desde Dentro, Universidad de Barcelona, Disponible en: http://diposit.ub.edu/dspace/bitstream/2445/41493/6/05.VLS_ANALISIS_Y_TRA TAMIENTO INFORMACION.[pdf]. 